\title{
Ultrasound-Enhanced Anodic Stripping Voltammetry Using Perfluorosulfonated Ionomer-Coated Mercury Thin-Film Electrodes
}

\author{
Frank-Michael Matysik, Silke Matysik, Ana Maria Oliveira Brett,* and Christopher M. A. Brett* \\ Departamento de Química, Universidade de Coimbra, 3049 Coimbra, Portugal
}

\begin{abstract}
The high mass transport caused by ultrasonic radiation in the preconcentration of trace metal species in anodic stripping voltammetry (ASV), leads to an order of magnitude enhancement in sensitivity. A Nafion-coated mercury thin-film working electrode is placed in a specially designed small-volume sonovoltammetric cell. The preparation and curing procedure for the Nafion film on a glassy carbon electrode substrate as well as ultrasound-enhanced mercury deposition through the Nafion film are optimized. The resulting electrode assembly is stable under ultrasonic irradiation. Optical microscopy observations show the formation of closely spaced mercury droplets of micrometer dimensions under the Nafion film. The performance characteristics of ultrasound-enhanced ASV at the Nafion-coated mercury thin-film electrode are studied in detail. The detection limit obtained for lead and cadmium test species is $3 \times 10^{-11} \mathrm{M}$ (30 s preconcentration in the presence of ultrasound). A relative standard deviation of $1.2 \%$ is obtained for repetitive determinations of $10 \mathrm{nM} \mathrm{Cd}^{2+}(\mathrm{n}=9)$.
\end{abstract}

Anodic stripping voltammetry (ASV) is well established as a powerful method for trace metal analysis. ${ }^{1}$ Its inherent sensitivity results from preconcentrating the metal of interest into or onto an electrode followed by an anodic stripping step, usually using a differential pulse or square wave voltammetric scan.

Potentially, the detection limit can be lowered via more efficient preconcentration. In other words, the higher the rate of mass transport during the deposition period the lower the attainable limit of detection. However, other prerequisites such as reproducibility of mass transport and stability of the electrode assembly under the preconcentration conditions have to be fulfilled. Several effective strategies have been described for preconcentrating the analyte in stripping voltammetric determinations. These include hydrodynamic systems such as the rotating disk electrode ${ }^{2,3}$ and stationary electrodes in combination with flowing solutions, typically in wall-jet ${ }^{4-6}$ configurations. Frequently, stirring is also used to impose convection in the electrolyte solution. In the case of microelectrodes, it has been demonstrated that sufficiently

(1) Wang, J. Stripping Analysis: Principles, Instrumentation and Applications; VCH: Deerfield Beach, FL, 1985.

(2) Florence, T. M. J. Electroanal. Chem. 1970, 26, 293-298.

(3) Brihaye, C.; Duyckaerts, G. Anal. Chim. Acta 1982, 143, 111-120.

(4) Gunasingham, H.; Ang, K. P.; N go, C. C. Anal. Chem. 1985, 57, 505-508.

(5) Omanovic, D.; Peharec, Z.; M agjer, T.; Lovric, M .; Branica, M . Electroanalysis 1994, 6, 1029-1033.

(6) Brett, C. M. A.; Lima, J. L. F. C; Garcia, M. B. Q. Analyst 1994, 119, 12291233. effective preconcentration can be achieved in quiescent solution on the basis of (hemi)spherical diffusion. 7,8

Sonovoltammetry, i.e., the performance of voltammetry in the presence of ultrasound, has recently been established as a powerful tool for studying electrochemical processes. ${ }^{9-14}$ The attractive features of ultrasound-enhanced voltammetry are as follows: (i) Ultrasonic irradiation leads to very high mass transport of electroactive species and their products to and from the electrode surface. ${ }^{10,14}$ (ii) Ultrasound can influence the mechanism of chemical and electrochemical reactions via the action of highly reactive radicals such as $\cdot \mathrm{OH}$ and $\cdot \mathrm{H}$ formed during sonolysis of water. ${ }^{15,16}$ (iii) The adsorption of species involved in an electrochemical reaction can be reduced in the presence of ultrasound. ${ }^{17}$ (iv) UItrasonic irradiation can provide continuous in situ activation of the electrode surface. ${ }^{11,17}$

Among a variety of experimental strategies of introducing ultrasound into an electrochemical cell, direct-immersion sonicator probes are highly practicable. A suitable cell configuration contains the working electrode introduced from the bottom and placed directly below the tip of a vibrating titanium horn. One of the most attractive characteristics of this experimental configuration is the enormous enhancement of mass transport due to the high ultrasonic power intensity present in the vicinity of the electrode. Recently we have designed and evaluated a smallvolume cell, 1 order of magnitude smaller than previous sonoelectrochemical cell designs, ${ }^{10,11}$ in which the coupling of ultrasound and voltammetry is particularly suitable for analytical studies. ${ }^{18}$

The aim of the present work is to utilize the unique hydrodynamic characteristics that result from insonication of electrolyte solutions as an effective way to preconcentrate the analyte in anodic stripping voltammetry. A recent paper by Coury and co-

(7) Wehmeyer, K. R.; Wightman, R. M. Anal. Chem. 1985, 57, 1989-1993.

(8) M atysik, F.-M .; Gläser, P.; Werner, G. Fresenius J. Anal. Chem. 1994, 349, 646-649.

(9) M ason, T. J.; Lorimer, J. P.; Walton, D. J. Ultrasonics 1992, 28, 333-337. (10) Hagan, C. R. S.; Coury, L. A., Jr. Anal. Chem. 1994, 66, 399-405.

(11) Compton, R. G.; Eklund, J. C.; Page, S. D.; Sanders, G. H. W.; Booth, J. J. Phys. Chem. 1994, 98, 12410-12414.

(12) Klíma, J.; Bernard, C.; Degrand, C. J. Electroanal. Chem. 1994, 367, 297300.

(13) Reisse, J.; Francois, H.; Vandercammen, J.; Fabre, O.; Kirsch-de M esmaeker A.; M aerschalk, C; Delplancke, J.-L. Electrochim. Acta 1994, 39, 37-39.

(14) Walton, D. J.; Phull, S. S.; Chyla, A.; Lorimer, J. P.; M ason, T. J.; Burke, L. D.; M urphy, M.; Compton, R. G.; Eklund, J. C.; Page, S. D. J. Appl. Electrochem. 1995, 25, 1083-1090.

(15) Compton, R. G.; Eklund, J. C.; Page, S. D. J. Phys. Chem. 1995, 99, 42114214.

(16) Compton, R. G.; M atysik, F.-M . Electroanalysis 1996, 8, 218-222.

(17) Oliveira Brett, A. M.; M atysik, F.-M. B ioelectrochem. Bioenerg., in press.

(18) Oliveira Brett, A. M .; M atysik, F.-M. Electrochim. Acta, in press. 
workers ${ }^{19}$ also reported stripping voltammetric experiments in combination with ultrasound. However, the authors described sonochemical deposition of metal particles in organic suspensions onto a gold electrode followed by subsequent anodic stripping in a separate aqueous solution. In contrast to this, our investigations-formation of mercury thin-film and stripping voltammetry experiments-are performed entirely in the sonovoltammetric cell. This approach requires special attention regarding the stability of the working electrode, i.e., protecting the integrity of the mercury film, in the presence of ultrasound. Therefore, the preparation of perfluorosulfonated ionomer ( $\mathrm{N}$ afion)-coated mercury thin-film electrodes resistant to sonication and their characterization plays an important role in the present paper. The performance characteristics of the sonovoltammetric stripping procedure are studied and analytical utility is explored.

\section{EXPERIMENTAL SECTION}

Apparatus. Sonovoltammetric measurements were carried out in the cell arrangement illustrated in Figure 1. The jacketed glass cell, suitable for an electrolyte volume of $20 \mathrm{~mL}$, was constructed such that the working electrode surface was facing the tip of the sonic horn probe. The horn was connected to a tapered microtip ( $3 \mathrm{~mm}$ diameter) which was fabricated from highgrade titanium alloy. The circulating coolant was water thermostated in a constant-temperature bath $\left(25^{\circ} \mathrm{C}\right)$. Under optimized experimental conditions, the distance between the sonic horn and the working electrode was $6 \pm 1 \mathrm{~mm}$ and an ultrasound power intensity of $30 \pm 3 \mathrm{~W} \mathrm{~cm}-2$ (calibrated according to the procedure of $\mathrm{M}$ ason et $\mathrm{al}^{2}{ }^{20}$ ) was selected. The high-intensity ultrasonic processor was a M odel VC501 (Sonics \& M aterials Inc.) rated by the manufacturer at $500 \mathrm{~W}$ when driven at $20 \mathrm{kHz}$ frequency.

All voltammetric experiments were conducted using a PGSTAT 10 Autolab voltammetric analyzer (Eco Chemie, Utrecht, Netherlands).

M icroscopic studies were carried out with an Optiphot optical microscope (Nikon, Tokyo, Japan). Photographs were taken with Kodak ASA 25 black and white or color films.

Chemicals. Solutions were prepared from analytical grade chemical reagents and M illipore M illi-Q ultrapure water (resistivity $\geq 18 \mathrm{M} \Omega \mathrm{cm}$ ). Stock solutions of $10^{-3} \mathrm{M} \mathrm{Pb}^{2+}$ and $\mathrm{Cd}^{2+}$ were prepared and diluted on the day as appropriate. A 5\%solution of Nafion in low-weight alcohols (Aldrich) was diluted with ethanol ( $M$ erck, pro analysi) prior to application. Cellulose acetate (acetyl content 40\%) was obtained from Sigma. N,N-Dimethylformamide (DM F) was of analytical grade ( $99.5 \%$ and purchased from Fluka.

The Standard Reference M aterial (SRM 1643c) was prepared at the facilities of the U.S. Geological Survey, B ranch of Quality Assurance, Golden, $\mathrm{CO}$, and obtained from the National Institute of Standards and Technology (NIST), Gaithersburg, MD.

Procedures. Nafion-coated mercury thin-film electrodes (NCMTFE) for ultrasound-enhanced stripping voltammetry were prepared according to the following procedure. First, the $6 \mathrm{~mm}$ diameter glassy carbon electrode (GCE), a gift from Prof. G. Jenkins, Alabama A\&M University, Normal, AL, was polished using polishing foils with $0.3 \mu \mathrm{m}$ alumina particles (Ehret Datentechnik, Emmendingen, Germany), rinsed with M illi-Q water and allowed to dry. Nafion coating was achieved by applying $10 \mu \mathrm{L}$

(19) M adigan, N. A.; M urphy, T. J.; Fortune, J. M .; Hagan, C. R. S.; Coury, L. A., Jr. Anal. Chem. 1995, 67, 2781-2786.

(20) M ason, T. J.; Lorimer, J. P.; Bates, D. M. Ultrasonics 1992, 30, 40-42.
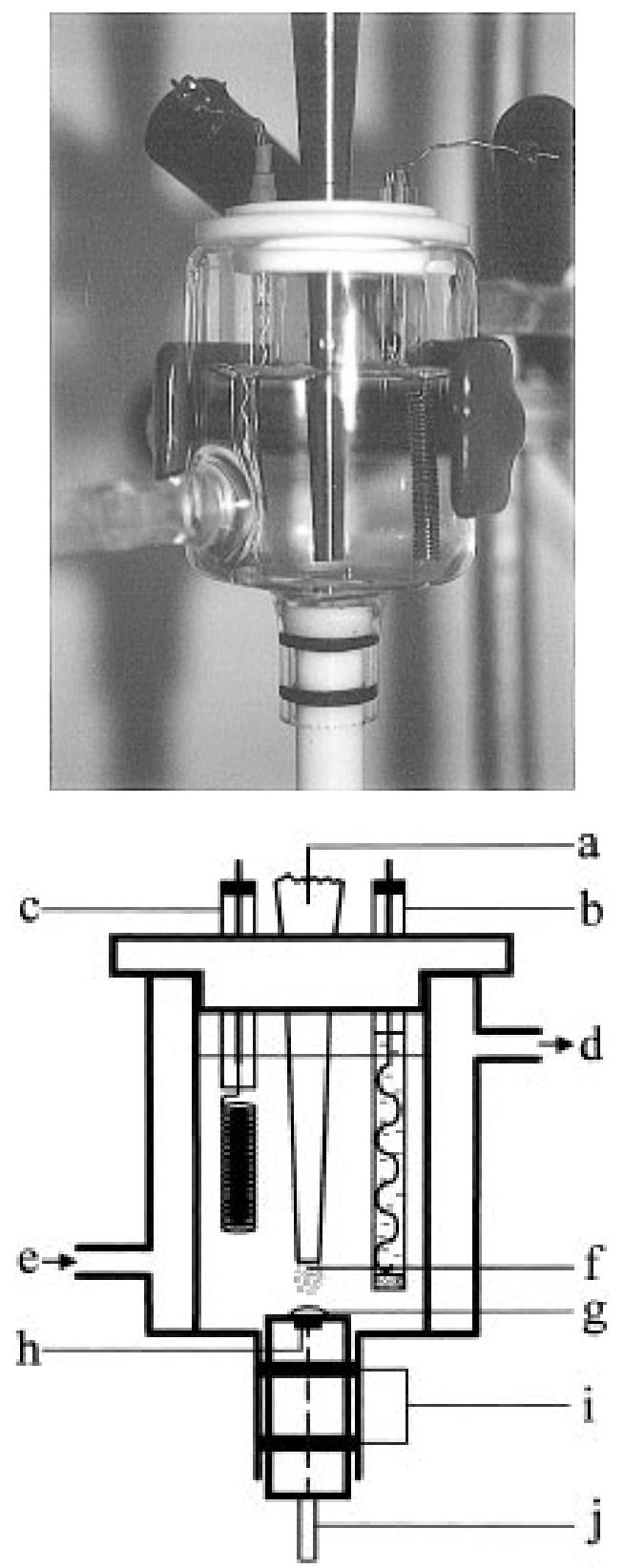

Figure 1. Photograph and schematic representation of the sonovoltammetric cell: a, sonic horn with microtip; b, $\mathrm{Ag} / \mathrm{AgCl}(3 \mathrm{M} \mathrm{KCl})$ reference electrode; $c$, platinum coil counter electrode; $d$, coolant outlet; e, coolant inlet; $f$, cavitational plume; g, Nafion-coated mercury film; h, glassy carbon; i, O-ring seal; j, working electrode lead.

of $0.5 \%(w / v)$ freshly prepared Nafion solution, followed immediately by $5 \mu \mathrm{L}$ of DM F casting solvent, to the electrode surface with a micropipet. The solvents were evaporated in a warm airstream from an air gun while the electrode was rotated at 50 rpm. The polymer film was then cured for $60 \mathrm{~s}$ in a hot airstream $\left(\sim 70{ }^{\circ} \mathrm{C}\right)$, holding the gun just a few millimeters above the electrode surface. The N afion-coated electrode was then placed in the sonovoltammetric cell containing $20 \mathrm{~mL}$ of $0.1 \mathrm{M}$ acetate buffer ( $\mathrm{pH} 4.60$ ), and three cyclic voltammograms were performed in the potential range between -1.0 and $0.0 \mathrm{~V}$ to verify a low background current. M ercury deposition was done by adding 40 $\mu \mathrm{L}$ of a solution of $0.1 \mathrm{M} \mathrm{Hg}^{2+}$ in $0.1 \mathrm{M} \mathrm{KNO}_{3} / 0.002 \mathrm{M} \mathrm{HNO}_{3}$ to the actetate buffer solution and applying a similiar potential program as in square wave anodic stripping voltammetry, as specified below, utilizing ultrasonic enhancement of the deposition. 


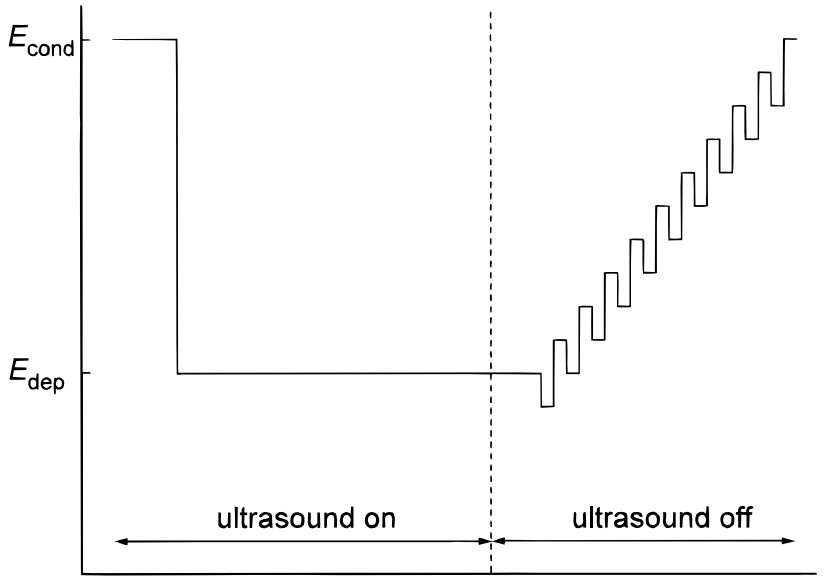

Time

Figure 2. Potential-timewave form for ultrasound-enhanced SWASV. Preconcentration time varied between 30 and $120 \mathrm{~s}$; ultrasound switched on $10 \mathrm{~s}$ before deposition period and switched off $5 \mathrm{~s}$ before end of deposition period. $E_{\text {cond }}$ and $E_{\text {dep }}$ represent the conditioning and deposition potentials, which are set at 0.0 and $-1.25 \mathrm{~V}$, respectively.

The NCM TFE was then ready for use in ASV measurements. The studies that led to this procedure are described in the next section.

The ultrasound-enhanced ASV procedure is illustrated in Figure 2. Experiments were undertaken without deoxygenation and the square wave parameters were as follows: amplitude 50 $\mathrm{mV}$, scan increment $2 \mathrm{mV}$, and frequency $50 \mathrm{~Hz}$. The conditioning potential, $\mathrm{E}_{\text {cond, was }} 0 \mathrm{~V}$ and the deposition potential, $\mathrm{E}_{\text {dep, was }}$ $-1.25 \mathrm{~V}$, which was also maintained under silent conditions for 5 $\mathrm{s}$ before starting the square wave scan. If not otherwise stated, the deposition time in the presence of ultrasound was $30 \mathrm{~s}$.

\section{RESULTS AND DISCUSSION}

Optimization of Preparing Nafion-Coated Mercury Thin-

Film Electrodes. The stability of the thin mercury film under conditions of ultrasonic irradiation is the most important point for performing reliable ultrasound-enhanced ASV measurements. Several procedures for preparing mercury thin films with and without polymer coating were examined. It was found that mercury thin films formed onto glassy carbon electrodes without an additional polymer coating were not suitable for sonovoltammetric studies in the present cell configuration. This is not surprising because mercury shows only weak interactions with the glassy carbon surface ${ }^{21}$ and the capability of high-intensity ultrasound to erode electrode material ${ }^{11,22}$ is well-known. Consequently, further studies were directed to polymer-coated mercury films.

Glassy carbon electrodes coated with cellulose acetate (prepared according to a procedure described in the literature ${ }^{23}$ ) or Nafion films were placed in the sonovoltammetric cell, and their stability in the presence of ultrasound was tested without mercury deposition. The adhesion of the cellulose acetate coatings on the glassy carbon substrate was not strong enough to be resistant to sonication. As a result, the whole film lifted off after sonication periods of less than 1 min.

(21) Frenzel, W. Anal. Chim. Acta 1993, 273, 123-137.

(22) M adigan, N. A.; Hagan, C. R. S.; Coury, L. A., Jr. J. Electrochem. Soc. 1994, 141, L23-L24.

(23) Dam, M . E. R.; Thomsen, K. N.; Pickup, P. G.; Schroder, K. H. Electroanalysis 1995, 7, 70-78.
The mechanical properties of Nafion films were found to be much better than those of cellulose acetate films. Nafion-coated electrodes were optimized in a similar way as described recently for the NCM TFE used in conjunction with batch injection analysis $(B \mid A),{ }^{24}$ in which volumes in the microliter range are injected perpendicularly from a micropipet onto the center of the electrode. The use of DMF as casting solvent and heating during film formation improved the stability of the Nafion films under conditions of ultrasonic irradiation. In addition, preparation by spin-coating at a rotation speed of $50 \mathrm{rpm}$ resulted in a more homogeneous polymer layer than under stationary conditions. Visually, the Nafion coating appeared transparent and uniform over the whole electrode surface. A typical optical micrograph of a Nafion-coated GCE is shown in Figure $3 \mathrm{~A}$, which also illustrates the roughness of the underlying glassy carbon surface.

An appropriate procedure for in situ mercury deposition through the Nafion film was then elaborated. Initially the mercury thin film was formed using the BIA principle and the optimized procedure described in ref 24 . To this end, $10 \mu \mathrm{L}$ of $0.1 \mathrm{M} \mathrm{Hg}^{2+}$ in $0.1 \mathrm{M} \mathrm{KNO}_{3} / 0.002 \mathrm{M} \mathrm{HNO}_{3}$ electrolyte solution was injected by micropipet on the top of the Nafion-coated GCE while a potential of $-1.0 \mathrm{~V}$ was applied. Such a NCM TFE is stable under the hydrodynamic conditions of BIA and corresponding stripping voltammetric determinations. ${ }^{24}$ However, in the presence of ultrasound this stability was not sufficient-after a few seconds of ultrasonic irradiation the entire film or parts of it were lifted off. Lower concentrations of $\mathrm{Hg}^{2+}$ were tried but led to insufficient mercury film formation since microliter volumes are employed in BIA mercury deposition.

A much more suitable approach was found to be the in situ deposition of mercury from an acetate buffer solution containing $\mathrm{Hg}^{2+}$, in the range of concentration normally used for mercury film formation on bare glassy carbon, in the presence of ultrasound. The potential program used was that of the square wave ASV (SWASV) procedure described in the Experimental Section, which involved a $30 \mathrm{~s}$ deposition period under ultrasonic irradiation; see Figure 2. High $\mathrm{Hg}^{2+}$ concentrations in the $20 \mathrm{~mL}$ sonovoltammetric cell led to too rapid film formation and lifting off of the Nafion from the substrate; experiments showed that a concentration of $2 \times 10^{-4} \mathrm{M} \mathrm{Hg}^{2+}$ led to a good response. A stable background response was obtained with this electrode after the first or second scan, and the NCM TFE was then ready for ASV determinations. Trace metal determinations could be performed in the same solution by adding an appropriate amount of the analyte sample to the $\mathrm{Hg}^{2+}$ / acetate buffer solution. There was good signal stability for repetitive measurements, to be quantified later, and the optical appearance of the N afion/ mercury assembly remained the same. Optical micrographs indicate that in representative central regions of the electrode small droplets of submicrometer size are formed (see Figure 3B). At the edge of the electrode, larger droplets can also be found as shown in Figure $3 C$ and $D$, possibly due to the inhomogeneity of the ultrasonic field. The tendency for droplet formation has been observed previously for the widely used mercury "films" deposited on bare glassy carbon substrates, e.g., refs 21 and 25, where the formation of truly continuous thin films does not occur in practice. The electroanalytical behavior is little affected if procedures suggested

(24) Brett, C. M . A.; Oliveira Brett, A. M .; M atysik, F.-M .; M atysik, S.; Kumbhat, S. Talanta 1996, 43, 2015-2022.

(25) Stulikova, M. J. Electroanal. Chem. 1973, 48, 33-45. 

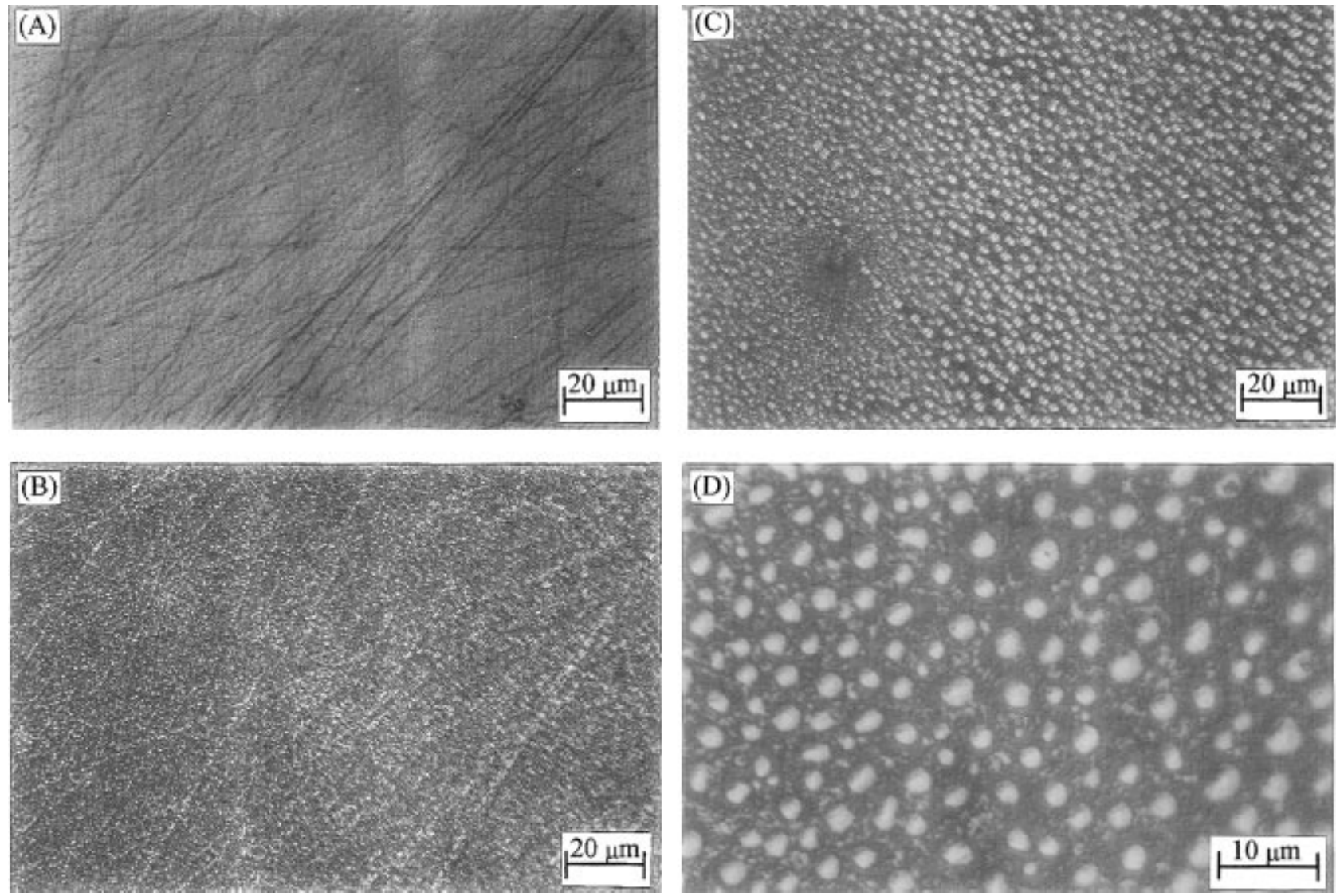

Figure 3. Optical micrographs of (A) Nafion coating on glassy carbon substrate, (B) Nafion-coated mercury thin film electrode homogeneous inner region; (C) Nafion-coated mercury thin film electrode, edge of electrode; and (D) Nafion-coated mercury thin film electrode, edge of electrode.

in the literature are followed.

The mercury droplets are embedded in a compact, transparent Nafion matrix. Focusing the optical microscope through the film confirms that the droplets are formed on the glassy carbon substrate beneath the Nafion film. The thickness of this film can be estimated using data for the density of bulk Nafion ${ }^{23}$ to be of the order of $1 \mu \mathrm{m}$. Thus the film is probably slightly lifted above where the drops form. Nevertheless, the ease of diffusion through such a thin film as demonstrated by the lack of limiting effects in square wave scans means that, effectively, linear diffusion still occurs.

Optical micrographs were also taken from NCM TFE prepared using mercury deposition by BIA. These NCM TFE showed more closely arranged, larger droplets than found for the NCMTFE prepared in the presence of ultrasound. Thus, and in accordance with the above, the BIA-prepared Nafion film will be adherent over a lesser fraction of the substrate due to these larger droplets on the substrate and hence will be more prone to lift off. Additionally, it can be speculated that the greater mass of mercury in each droplet is also responsible for instability in the presence of ultrasound because the potential to cause mechanical destruction should be mass-dependent. For this reason, all of the following ASV experiments were done with NCM TFEs prepared by in situ deposition of mercury through the Nafion film as described in the Experimental Section.

The ultrasound conditions, i.e., the horn tip-electrode separation and the power intensity, were also varied. The chosen parameters (horn tip-electrode separation $6 \mathrm{~mm}$, power intensity $30 \mathrm{~W} \mathrm{~cm}^{-2}$ ) are a compromise between achieving high mass transport and ensuring stability of the NCM TFE. Under these

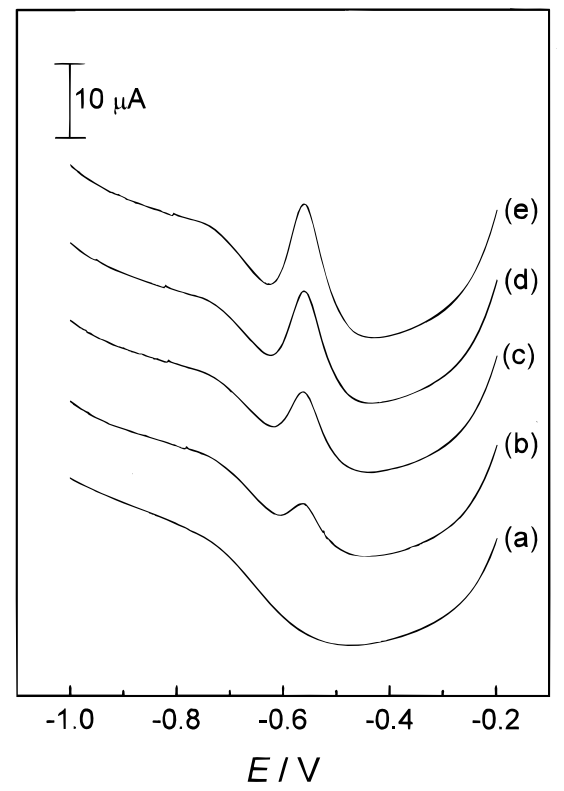

Figure 4. Ultrasound-enhanced SWASV of $5 \mathrm{nM} \mathrm{Pb}^{2+}$ at Nafioncoated mercury thin film electrode. Deposition times: (a) 30 (no ultrasound), (b) 30, (c) 60, (d) 90, and (e) $120 \mathrm{~s}$.

conditions the NCMTFE could be used for two days (4-5 h per day) without changes in the performance characteristics, whereas on the third day there was usually a decrease in sensitivity caused by the appearance of flaws in the Nafion coating. The NCMTFE was stored in air between experiments.

Analytical Performance Characteristics. (i) Sensitivity and Limit of Detection. Figure 4 shows the SWASV response 


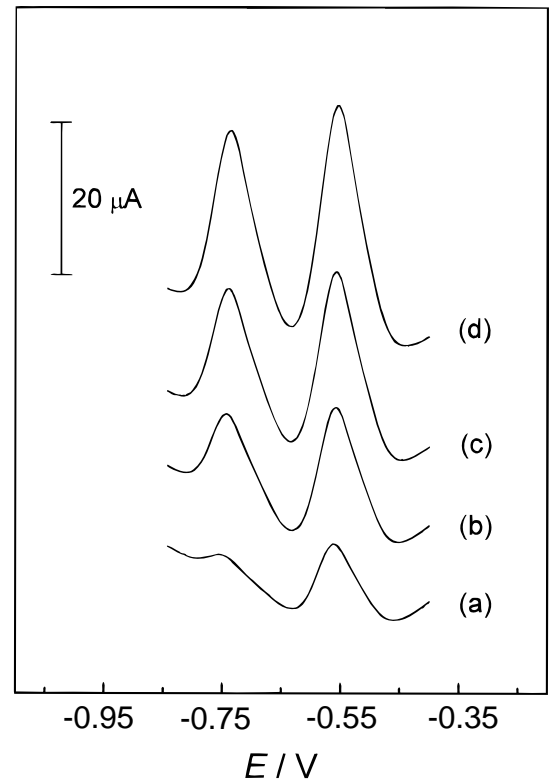

Figure 5. Ultrasound-enhanced SWASV of $\mathrm{Cd}$ and $\mathrm{Pb}$ at Nafioncoated mercury thin-film electrode. Concentrations: (a) $\left[\mathrm{Cd}^{2+}\right]=2$ $\mathrm{nM},\left[\mathrm{Pb}^{2+}\right]=7 \mathrm{nM}$; (b) $\left[\mathrm{Cd}^{2+}\right]=7 \mathrm{nM},\left[\mathrm{Pb}^{2+}\right]=12 \mathrm{nM}$; (c) $\left[\mathrm{Cd}^{2+}\right]=$ $12 \mathrm{nM},\left[\mathrm{Pb}^{2+}\right]=17 \mathrm{nM}$; (d) $\left[\mathrm{Cd}^{2+}\right]=17 \mathrm{nM},\left[\mathrm{Pb}^{2+}\right]=22 \mathrm{nM}$. Preconcentration for $30 \mathrm{~s}$ in the presence of ultrasound.

Table 1. Linear Regression of Calibration Data for Cadmium and Lead Determination by UltrasoundEnhanced SWASV Using a Nafion-Coated Mercury Thin-Film Electrode ${ }^{a}$

\begin{tabular}{cccc} 
analyte & $\begin{array}{c}\text { slope } \pm \\
\mathrm{Cd}^{2+}\end{array}$ & $\begin{array}{c}\text { intercept } \pm \\
\sigma(\mu \mathrm{A} / \mathrm{nM})\end{array}$ & $\begin{array}{c}\text { regression } \\
\text { coeff }\end{array}$ \\
$\mathrm{Pb}^{2+}$ & $1.35 \pm 0.03$ & $0.08 \pm 0.30$ & $0.9979(\mathrm{n}=9)$ \\
& $1.21 \pm 0.02$ & $0.14 \pm 0.60$ & $0.9991(\mathrm{n}=7)$ \\
a Deposition time 30 s. & & \\
\hline
\end{tabular}

obtained for the determination of $5 \mathrm{nM} \mathrm{Pb}^{2+}$ using different preconcentration conditions: silent and ultrasound-enhanced. Increasing the deposition time with ultrasound-enhanced deposition in the range $30-120 \mathrm{~s}$ leads to a linear increase in the ASV signal. It should be emphasized that curve a represents not just a background response but was obtained after a deposition period of $30 \mathrm{~s}$ under silent conditions for which the lead concentration tested is obviously below the detection limit.

Comparison was also made with preconcentration in which the solution was agitated by magnetic stirring. It was found that the preconcentration efficiency in the presence of ultrasound was $\sim 5$ times higher than that under magnetic stirring at the highest possible speed ( $\sim 500 \mathrm{rpm})$. As a consequence of the enhanced preconcentration due to ultrasound, quite short deposition times of $30 \mathrm{~s}$ were usually selected for ultrasound-assisted ASV measurements.

Figure 5 illustrates the typical response obtained for the test species lead and cadmium present at concentrations in the lower nanomolar range. Calibration plots were linear within the concentration range from 5 to $50 \mathrm{nM}$; the results of linear regression are shown in Table 1. Higher concentrations can be measured but memory effects begin to appear-it is therefore advisable to dilute as appropriate.

Detection limits were determined on the basis of the $3 \sigma$ criterion where $\sigma$ represents the standard deviation of the baseline
Table 2. Analysis of a Standard Reference Material by Ultrasound-Enhanced ASV. Comparison between Certified Values and Experimental Results

\begin{tabular}{ccc} 
element & $\begin{array}{c}\text { certified concn } \\
(\mathrm{mg} / \mathrm{mL})( \pm 2 \sigma)\end{array}$ & $\begin{array}{c}\text { results obtained by US-ASV } \\
(\mathrm{mg} / \mathrm{mL})( \pm 2 \sigma)\end{array}$ \\
$\mathrm{Cd}$ & $12.2 \pm 1.0$ & $11.4 \pm 1.6$ \\
$\mathrm{~Pb}$ & $35.3 \pm 0.9$ & $38.6 \pm 3.2$ \\
\hline
\end{tabular}

noise. The limit of detection ( $30 \mathrm{~s}$ preconcentration) is $3 \times 10^{-11}$ $M$ for both cadmium and lead. However, in practice the detection limit was $\sim 1$ magnitude of order higher because trace metal impurities were encountered in the chemicals employed.

(ii) Precision and Accuracy. The reproducibility of repetitive ultrasound-enhanced ASV experiments was characterized by performing successive determinations of $10 \mathrm{nM} \mathrm{Cd}^{2+}$. As already mentioned, the in situ deposition of mercury requires one or two ASV determinations to establish stable electrode response. Thus, for statistical evaluation, the first measurement was not included. A relative standard deviation of $1.2 \%$ was obtained.

Signal stability was also studied in the case of electrolyte exchange, i.e., after mercury deposition the cell electrolyte was replaced by an acetate buffer not containing $\mathrm{Hg}^{2+}$. As a result,

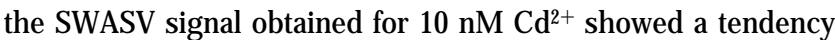
to decrease slightly in successive experiments (between 3 and $7 \%$ after 10 measurements). Thus, most experiments were conducted in the presence of $\mathrm{Hg}^{2+}$. However, care has to be taken because commercially available mercury(II) salts often contain heavy metal impurities which can lead to blank values in the lower nanomolar range.

Finally, the accuracy of ultrasound-enhanced ASV was examined. SRM 1643c (see Experimental Section), an aqueous solution containing 27 elements, was analyzed with respect to lead and cadmium content. The reference material was diluted by a factor of 20 , and the determinations were done by the standard addition method. Comparison between the certified concentrations and those obtained with ultrasound-enhanced SWASV is shown in Table 2. The results obtained agree well with the certified values, taking the statistical uncertainty into consideration. The standard deviations given in Table 2 are significantly higher than those obtained for repetitive measurements with the same NCMTFE and without changing the electrolyte solution (see results above). Thus, the reproducibility has also to be associated with the preparation of the individual NCMTFEs and the handling of analyte solutions.

\section{CONCLUSIONS}

Ultrasound-enhanced SWASV has been demonstrated to be a reliable and accurate analytical method for highly sensitive trace metal determinations. The main effect of ultrasound is greatly enhanced mass transport, which can be exploited for effective preconcentration of analyte species in ASV. The in situ deposition of mercury through a Nafion film has resulted in a reliable electrode assembly which is resistant to ultrasonic irradiation.

For practical applications, the Nafion coating has the additional advantage of reducing interferences from complex matrices. In particular, it appears to be attractive to direct future studies of ultrasound-enhanced ASV toward biological and environmental samples by utilizing other effects of ultrasound such as rapid cell disruption and homogenization of sample material. This approach 
could lead to further simplification of "real-life" trace metal analysis with rapid response characteristics and a lesser risk of contamination in comparison with procedures involving separate sample pretreatment or digestion steps.

\section{ACKNOWLEDGMENT}

We thank the EU Human Capital and Mobility Programme for support under Contract CHRXCT 940475 and J unta Nacional de Investigação Científica e Tecnológica (J NICT), Portugal, under Contract PEAM / P/ SEL/ 526/ 95.

Received for review August 12, 1996. Accepted December 8, 1996. ${ }^{\otimes}$

\section{AC960815J}

${ }^{\otimes}$ Abstract published in Advance ACS Abstracts, M arch 1, 1997. 